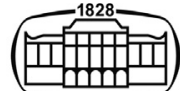

AKADÉMIAI KIADÓ

Journal of Behavioral Addictions

10 (2021) 1, 99-111

DOI:

$10.1556 / 2006.2021 .00003$

(c) 2021 The Author(s)

\section{FULL-LENGTH REPORT}

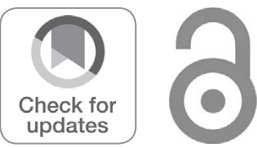

\title{
Enhanced neural responses in specific phases of reward processing in individuals with Internet gaming disorder
}

\section{LINGXIAO WANG ${ }^{1,2,3,4}$, GUOCHUN YANG $^{5,6}$, YA ZHENG ${ }^{7}$, ZHENGHAN LI ${ }^{5,6}$, YUE QI ${ }^{8,9}$, QI LI ${ }^{1,5,6 *} \odot$ and XUN LIU ${ }^{5,6}$}

\author{
${ }^{1}$ Department of Psychology, Beijing Key Laboratory of Learning and Cognition, Capital Normal \\ University, Beijing, China \\ ${ }^{2}$ Center for Cognition and Brain Disorders, The Affiliated Hospital of Hangzhou Normal University, \\ Hangzhou, Zhejiang Province, China \\ ${ }^{3}$ Institutes of Psychological Sciences, Hangzhou Normal University, Hangzhou, China \\ ${ }^{4}$ Zhejiang Key Laboratory for Research in Assessment of Cognitive Impairments, Hangzhou, \\ Zhejiang Province, China \\ ${ }^{5}$ CAS Key Laboratory of Behavioral Science, Institute of Psychology, Beijing, China \\ ${ }^{6}$ Department of Psychology, University of Chinese Academy of Sciences, Beijing, China \\ ${ }^{7}$ Department of Psychology, Dalian Medical University, Dalian, China \\ ${ }^{8}$ The Department of Psychology, Renmin University of China, Beijing, China \\ ${ }^{9}$ The Laboratory of the Department of Psychology, Renmin University of China, Beijing, China
}

Received: August 11, 2020 • Revised manuscript received: November 25, 2020 - Accepted: December 27, 2020

Published online: February 10, 2021

\begin{abstract}
Background and aims: Internet gaming disorder (IGD) has become a global health problem. The selfregulation model noted that a shift to reward system, whether due to overwhelming reward-seeking or impaired control, can lead to self-regulation failures, e.g., addiction. The present study focused on the reward processing of IGD, aiming to provide insights into the etiology of IGD. Reward processing includes three phases: reward anticipation, outcome monitoring and choice evaluation. However, it is not clear which phases of reward processing are different between individuals with IGD and healthy controls (HC). Methods: To address this issue, the present study asked 27 individuals with IGD and 26 $\mathrm{HC}$ to complete a roulette task during a functional MRI scan. Results: Compared with HC, individuals with IGD preferred to take risks in pursuit of high rewards behaviorally and showed exaggerated brain activity in the striatum (nucleus accumbens and caudate) during the reward anticipation and outcome monitoring but not during the choice evaluation. Discussion: These results reveal that the oversensitivity of the reward system to potential and positive rewards in college students with IGD drives them to approach risky options more frequently although they are able to assess the risk values of options and the correctness of decisions properly as HC do. Conclusions: These findings provide partial support for the application of the self-regulation model to the IGD population. Moreover, this study enriches this model from the perspective of three phases of reward processing and provides specific targets for future research regarding effective treatment of IGD.
\end{abstract}

\section{KEYWORDS}

fMRI, Internet gaming disorder, reward processing

\section{INTRODUCTION}

With the increasing development of the Internet in the past decades, online gaming has become a very popular leisure activity, especially among college students. According to the 
("Report on China's game industry from January to June 2019”, 2019), there are 640 million gamers in China alone. However, excessive gaming behavior can have various negative consequences, such as poor school/work performance, sleep insufficiency, and heightened anxiety levels (Kuss \& Griffiths, 2012; Petry et al., 2014). Gamers who persistently and uncontrollably engage in online gaming despite these negative consequences are considered to suffer from Internet gaming disorder (IGD) (Petry et al., 2014). In view of the significant prevalence and acute impacts of IGD, it was first included as a tentative disorder in the fifth version of Diagnostic and Statistical Manual of Mental Disorders (DSM-5) (APA, 2013) and then officially included as an addictive disorder in the eleventh version of International Classification of Diseases (ICD-11) (https://icd.who.int/ browse11/l-m/en). Therefore, there is an urgent need to investigate the mechanism of IGD to advance the treatment and prevention of this disorder.

Based on multiple neuroimaging studies, the prefrontalsubcortical model of self-regulation proposed by (Heatherton \& Wagner, 2011) noted that a shift in favor of the subcortical reward system, whether due to an overwhelming bottom-up reward-seeking or impaired top-down control function, can lead to self-regulation failures, e.g., addiction. Numerous studies emphasized the crucial role of the reward system in understanding addictive behaviors, including relapse and craving (Brand, Young, Laier, Wölfling, \& Potenza, 2016; Cooper, Robison, \& Mazei-Robison, 2017; Gardner, 2011; Volkow, Wang, Fowler, Tomasi, \& Telang, 2011). Thus, the present study focused on the reward processing of IGD, aiming to provide insights into the etiology of IGD. Several studies using task-state functional MRI (fMRI) have established that individuals with IGD show enhanced rewardseeking behavior and dysfunctional neural substrates related to reward processing. Some of these studies focused on the differences in how individuals with IGD and healthy controls (HC) responded to addiction-related cues, such as gamingrelated pictures or videos. Compared with HC, individuals with IGD showed an increased desire to play games and hyperactivation of brain regions crucial to craving and reward processing (e.g., the striatum, orbitofrontal cortex (OFC)) when presented with gaming-related cues (Ko et al., 2009; Liu et al., 2017; Wang et al., 2017).

However, other studies focused on the differences between individuals with IGD and HC in specific phases of reward processing, e.g., reward anticipation/decision and outcome monitoring. Reward anticipation is classified as the period during which participants are pondering potential choices before making a decision; for example, choosing a bet and expecting to win money would be classified as positive anticipation (Cohen \& Ranganath, 2005). Outcome monitoring is defined as the period during which participants receive feedback on a decision, such as via a screen indicating that they win or lose a bet (Bjork et al., 2004). Based on a meta-analysis of 142 neuroimaging studies on brain activation during reward-related tasks in healthy adults (Liu, Hairston, Schrier, \& Fan, 2011), the striatum (e.g., nucleus accumbens (NAcc) and caudate) is found to respond to general reward processing, which is a core region across the two phases of reward processing. The anterior cingulate cortex (ACC) and insula are implicated in risk uncertainty assessment in the anticipation phase. Additionally, the OFC is mostly activated by the delivery of outcomes. Compared with HC, individuals with Internet addiction exhibited increased activation in the caudate when they were choosing risky options for high rewards in the reward anticipation phase of a decision-making task (Seok, Lee, Sohn, \& Sohn, 2015). In the outcome monitoring phase, individuals with IGD exhibited higher activity than $\mathrm{HC}$ in the OFC and striatum when facing positive outcomes in a card-guessing task, demonstrating enhanced reward sensitivity in IGD (Dong, Huang, \& Du, 2011; Dong, Li, Wang, \& Potenza, 2017).

According to X. Liu and colleagues, in addition to these two phases, reward processing has a third stage: choice evaluation (Liu et al., 2007). Choice evaluation is defined as the period during which participants evaluate whether their choices are right or wrong, which is determined by the combination of the reward anticipation and outcome monitoring phases. It includes two conditions. One is considered a right choice, in which people choose a bet and win money or choose not to bet and avoid a loss, whereas the other is considered a wrong choice, in which people choose a bet and lose money or choose not to bet and miss a win. The case of choosing a bet and losing money is commonly known as a reward prediction error (PE) (Liu et al., 2007).

PEs measure deviations between expected outcomes and received outcomes (e.g., expecting a win but receiving a loss) (Schultz, Dayan, \& Montague, 1997). Converging evidence shows that the midbrain dopamine regions, striatum and prefrontal cortex (e.g., dorsolateral prefrontal cortex (DLPFC) and OFC) play major roles in coding PE (Cohen, 2006; Hare, O"Doherty, Camerer, Schultz, \& Rangel, 2008; Ramnani, Elliott, Athwal, \& Passingham, 2004; Schultz, 2004). Research on addiction has shown that the expression of striatal PEs in alcohol-dependent patients was intact; however, their functional connectivity between the striatum and DLPFC was abnormal, and this abnormality significantly predicted their alcohol craving and impairments in learning (Park et al., 2010). Another study also showed a weaker association between PEs and DLPFC activity in abstinent alcohol-dependent patients than in HC (Beylergil et al., 2017). Additionally, a reduced association between PEs and activity in the ventral striatum and medial OFC was found in abstinent substancedependent patients (Tanabe et al., 2013). These findings suggest an abnormal frontal-striatal circuit in substance addicts when processing $\mathrm{PE}$. Moreover, according to the findings in $\mathrm{HC}$ of (Liu et al., 2007), in addition to the striatum, OFC and DLPFC, the choice evaluation also recruits the insula.

Choice evaluation helps guide individual future decisionmaking behavior, which is crucial in addictive disorders. However, the neural substrate of the choice evaluation phase in IGD is still unknown. Moreover, there are too few previous studies on the anticipation and outcome monitoring phases of IGD to make a relatively consistent conclusion. Accordingly, in the present study, by applying a gambling task, namely, the roulette task (Liu et al., 2007), we aimed to 
thoroughly and simultaneously investigate the neural underpinnings of all three phases of reward processing to identify which of these phases are different in IGD and thus provide promising specific targets for the treatment and prevention of IGD. In terms of the aforementioned findings on reward processing in IGD and HC, we hypothesized that IGD participants would have a stronger willingness to take risks to seek high rewards than $\mathrm{HC}$ and show enhanced neural responses in areas crucial to reward processing (e.g., striatum, OFC) relative to $\mathrm{HC}$, at least in the reward anticipation and outcome monitoring phases.

\section{METHODS}

\section{Participants}

Twenty-seven right-handed college students with IGD and twenty-six matched HC were recruited from local colleges by cyber propagation. No participants had any historical or current neurological/psychiatric disorders or other emotional disorders, e.g., depression and anxiety. They were free of other addictions, including substance abuse, alcohol and nicotine dependence. Young's Internet Addiction Test (IAT) (Young, 1998) and the diagnostic criteria proposed by the DSM-5 (Petry et al., 2014) were used in the present study to classify IGD and HC participants. The IAT has been validated as reliable in diagnosing Internet addiction (Lai et al., 2013; Pawlikowski, Altstötter-Gleich, \& Brand, 2013; Widyanto \& Mcmurran, 2004). It is a 20 -item test on a 5point scale (1-rarely, 5-always) that measures the degree of online Internet use-related issues, such as withdrawal, compulsive use, and other problems in work/school, sleep or time management. Self-report scores above 50 indicate occasional or frequent Internet use-related issues due to uncontrolled Internet use. Thus, we used 50 as the cutoff score for the diagnosis of IGD, which has been widely used in studies on IGD (Cai et al., 2016; Dong \& Potenza, 2016; Dong, Wang, Wang, Du, \& Potenza, 2019; Xing et al., 2014). In addition, the DSM-5 criteria for IGD contain nine criteria, and each criterion depicts one Internet gaming userelated symptom, such as enhanced tolerance to gaming and abstinence syndrome. Participants were asked to indicate whether they met these criteria, as affirmed by the clinical interview by an experienced psychiatrist. Referring to the study by Petry et al. (2014) and previous studies (Ko et al., 2014; Wang et al., 2017; Yao et al., 2017; Yuan et al., 2016), a cutoff point of meeting five criteria in the DSM-5 was proposed for the diagnosis of IGD.

Accordingly, IGD participants were screened based on the following criteria: (1) had an IAT $\geq 50$; (2) met five or more DSM-5 criteria; (3) spent most of their Internet time on online gaming; and (4) played online games more than 21 hours per week, with a gaming history of at least 2 years. The inclusion criteria for HC participants were as follows: (1) barely played online games; (2) had an IAT $\leq 30$; and (3) did not meet any DSM-5 criteria. As shown in Table 1, the IGD and HC groups were matched by age and education level. The IGD group reported significantly longer online gaming time and higher IAT and DSM-5 scores than the HC group. They were asked not to drink any coffee or tea during the scanning day.

\section{Measures}

The timeline of one trial in the roulette task is shown in Fig. 1. In the first slide (the reward anticipation phase), participants were instructed to decide between a safe wheel and a risky wheel within $4 \mathrm{~s}$ to indicate whether to bet a certain number of chips. The wager of each trial is the number shown on the safe wheel. The initial wager was 3 chips. The safe wheel, shown in all green, represented banking the current wager with $100 \%$ probability. The green

Table 1. Demographic and behavioral characteristics of the two groups

\begin{tabular}{|c|c|c|c|c|}
\hline & IGD $(N=27)$ & $\mathrm{HC}(N=26)$ & $t$ & $P$ \\
\hline Age (years) & $22.52 \pm 2.33$ & $23.23 \pm 2.37$ & -1.10 & 0.275 \\
\hline Education (years) & $15.81 \pm 1.92$ & $16.35 \pm 2.65$ & -0.84 & 0.406 \\
\hline Years playing online games & $7.20 \pm 4.01$ & $0.53 \pm 0.85$ & 8.44 & $<0.001$ \\
\hline Gaming playing per week (hours) & $25.70 \pm 7.96$ & $0.33 \pm 0.83$ & 16.47 & $<0.001$ \\
\hline IAT scores & $70.15 \pm 8.53$ & $22.31 \pm 4.09$ & 26.18 & $<0.001$ \\
\hline DSM-V scores for IGD & $6.48 \pm 1.91$ & $0.23 \pm 0.65$ & 16.07 & $<0.001$ \\
\hline BAS/BIS BAS total & $42.04 \pm 4.12$ & $40.12 \pm 4.74$ & 1.58 & 0.121 \\
\hline BAS/BIS drive & $12.44 \pm 1.74$ & $12.38 \pm 2.08$ & 0.11 & 0.910 \\
\hline BAS/BIS fun seeking & $15.85 \pm 2.28$ & $14.15 \pm 2.56$ & 2.55 & $0.014^{*}$ \\
\hline BAS/BIS reward responsiveness & $13.74 \pm 1.70$ & $13.58 \pm 1.70$ & 0.35 & 0.727 \\
\hline BAS/BIS BIS & $16.52 \pm 2.24$ & $16.58 \pm 1.84$ & -0.10 & 0.918 \\
\hline SSS total & $17.67 \pm 6.64$ & $16.00 \pm 7.32$ & 0.87 & 0.389 \\
\hline SSS disinhibition & $3.93 \pm 2.15$ & $3.88 \pm 2.14$ & 0.07 & 0.944 \\
\hline SSS experience seeking & $4.48 \pm 2.08$ & $3.81 \pm 2.19$ & 1.15 & 0.256 \\
\hline SSS boredom susceptibility & $3.11 \pm 1.74$ & $2.50 \pm 1.90$ & 1.22 & 0.228 \\
\hline SSS thrill and adventure seeking & $6.15 \pm 2.66$ & $5.81 \pm 2.79$ & 0.46 & 0.651 \\
\hline
\end{tabular}

Table values: mean \pm standard deviation.

Abbreviations: IGD = Internet gaming disorder; HC = healthy controls; IAT $=$ Internet addiction test; DSM-V = The fifth version of Diagnostic and Statistical Manual of Mental Disorders; BAS/BIS = Behavioral Activation System/Behavioral Inhibition System; SSS = Sensation Seeking Scale. ${ }^{*} P<0.05$. 


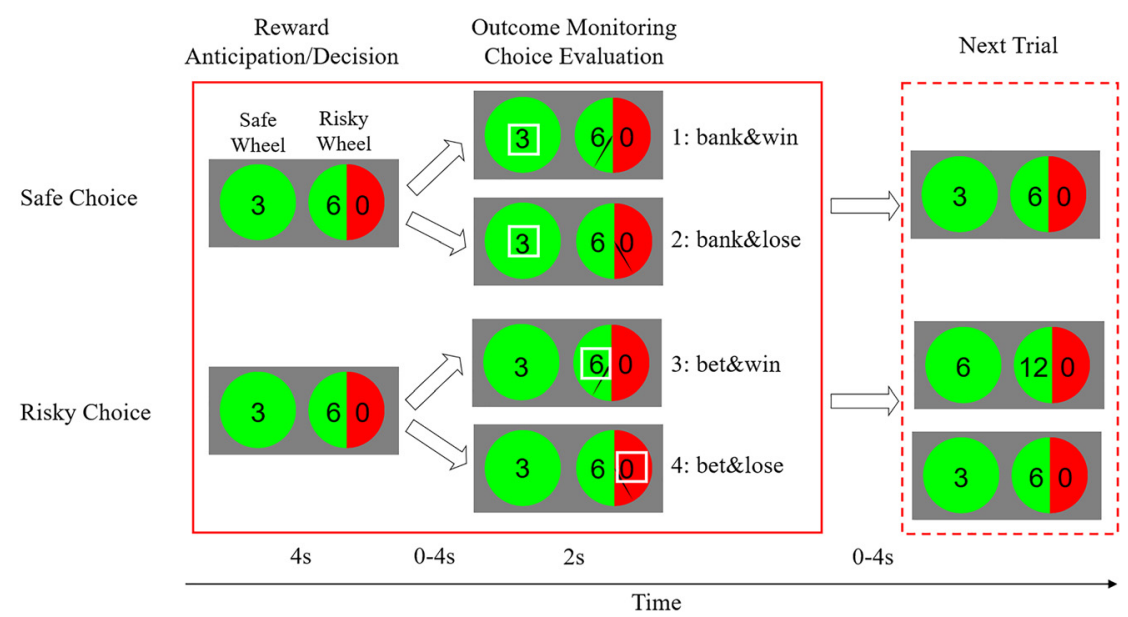

Fig. 1. Timeline of the roulette task. This task contains three phases of reward processing: reward anticipation/decision in the first slide and outcome monitoring and choice evaluation in the second slide. As shown on the first slide, participants were instructed to decide between a safe wheel and a risky wheel to indicate whether to bet the amount of chips showed on the safe wheel. As shown on the second slide, participants were presented with the outcomes of both their chosen and unchosen wheels in the decision phase. This figure shows the four cases in terms of participants' choices and outcomes. The winning condition (cases 1 and 3 ) and losing condition (cases 2 and 4 ) constituted the outcome monitoring phase. The right condition (cases 2 and 3) and wrong condition (cases 1 and 4) constituted the choice evaluation phase. The winning chips would continue to be taken as the next wager if participants chose to bet and won. The wager would start again with 3 chips once participants chose to bank or chose to bet and lost in the previous trial. The winning probability of the risky wheel varied randomly among $25 \%, 50 \%$ and $75 \%$. Additionally, the locations of the two wheels were randomly alternated between the left and right.

part of the risky wheel represented the probability of winning the displayed amount of chips, including 25, 50, or $75 \%$, whereas the red part of the risky wheel indicated the probability of losing the current wager. The safe and risky wheels of each trial were matched in expected value (probability $\times$ magnitude) but differed in risk value. The risk value is calculated by the following formula: risk $=[(1-$ winning probability $) \times(0-\text { expected value })^{2}+$ winning

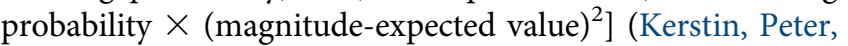
\& Quartz, 2006). For instance, considering a safe wheel with 3 chips and a risky wheel with 6 chips at $50 \%$ probability or 0 chips at $50 \%$ probability, the two wheels are equivalent in the expected value $(+3)$, but their risk values are different: safe wheel $=0$, risk wheel $=9$.

In the second slide (the outcome monitoring and choice evaluation phases), the outcomes of both the chosen wheel and the unchosen wheel in the decision phase were presented for $2 \mathrm{~s}$. A white box highlighted the outcome of the participants' chosen wheel. The outcome of the risky wheel was decided by the position at which the black needle stopped on the wheel. In fact, to ensure a balanced design, the outcomes of the risky wheels were predetermined, i.e., the proportions of winning and losing trials were proportional to the corresponding probabilities for each winning probability level. Figure 1 shows the four cases in terms of participants' choices and outcomes. (1) Bank and win and (2) Bank and lose: if participants chose the safe wheel, the current wager would be saved to their bank, and the next wager would be another 3 chips taken out from their bank. The outcome of the risky wheel would still be presented to participants. (3) Bet and win: if they chose the risky wheel and won, the winning chips would continue to be taken as the next wager, with one exception: if the current wager exceeded 100 chips, it would be banked, and the next trial would start over with 3 chips as the wager. (4) Bet and lose: if participants lost, the current wager would be expropriated, and another 3 chips would be taken from their bank as the next wager. "Outcome monitoring" refers to monitoring the feedback that indicates a win or a loss; thus, the winning conditions (cases 1 and 3) and losing conditions (cases 2 and 4) constituted this phase. "Choice evaluation" refers to evaluating whether a choice was right or wrong; thus, the right condition (cases 2 and 3 ) and the wrong condition (cases 1 and 4) constituted this phase.

In total, participants needed to play four runs of $4 \mathrm{~min}$ each under fMRI scanning. Each run comprised 24 trials, with eight trials at each winning probability level. Before each run, participants were offered 30 chips as the initial endowment and were encouraged to maximize their total chips as much as possible with various strategies. At the end of the task, accumulated chips of four runs were presented to participants, and one of the four runs was randomly selected as their bonus. Before entering the scanner, participants completed a practice version with 20 trials to familiarize themselves with the task.

\section{Procedure}

Imaging data acquisition. A GE 3-T (Discovery MR750) scanner equipped for echo-planar imaging (EPI) was used to collect the imaging data. The following parameters were used for fMRI acquisition: slice number $=37$, slice thickness $=3.5 \mathrm{~mm}$, interleaved sequence, repetition time $(\mathrm{TR})=2,000 \mathrm{~ms}$, echo time $(\mathrm{TE})=30 \mathrm{~ms}$, flip angle $=90^{\circ}$, field of view $(\mathrm{FOV})=224 \times 224 \mathrm{~mm}^{2}$ and voxel size $=$ $3.5 \times 3.5 \times 3.5 \mathrm{~mm}^{3}$. A computer running E-prime completed behavioral data acquisition and stimuli 
presentation. At the end of the experiment, a high-resolution 3D BRAVO T1-weighted anatomical set was acquired with 196 slices, $\mathrm{TR}=6.652 \mathrm{~ms}$, TE $=2.928 \mathrm{~ms}$, inversion time $(\mathrm{TI})=450 \mathrm{~ms}$, flip angle $=12^{\circ}, \mathrm{FOV}=256 \times 256 \mathrm{~mm}^{2}$ and voxel size $=1 \times 1 \times 1 \mathrm{~mm}^{3}$.

Questionnaires. Participants were instructed to complete two questionnaires after all fMRI scans: the behavioral activation system/behavioral inhibition system (BAS/BIS) and sensation-seeking scale (SSS). The BAS/BIS includes the BAS (three subscales: drive, fun seeking and reward responsiveness) and BIS scales, which measure participants' appetitive system and aversive system, respectively. A high BAS score indicates a strong desire for a reward, and a high BIS score indicates a large sensitivity to punishments (Carver \& White, 1994; Li et al., 2008). The SSS measures participants' sensation-seeking traits. It includes four subscales: disinhibition, experience seeking, boredom susceptibility and thrill and adventure seeking subscales. A high SSS score indicates an individual's tendency to take risks for novel and intense stimulation (Wang et al., 2000).

\section{Statistical analysis}

Behavioral data analysis. All analyses were performed after removing trials in which participants made no response within $4 \mathrm{~s}$ of the decision stage and collapsing remaining trials across different winning probabilities. First, two basic indexes, i.e., the proportion of bet and decision time, were compared between the IGD and HC groups using an independent-sample $t$-test. Second, two further indexes were analyzed to evaluate participants' risk preferences: (1) The condition in which participants chose to bet and won money 2 or 3 times in a row was defined as a winning streak. Due to the dynamic nature of the roulette task (i.e., the wager would add up if the participant chose to bet and won in the previous trial, as stated in the Measures of the METHODS section), the wager was accumulated and its magnitude was much larger after a winning streak. Therefore, the number of winning streaks and the proportion of bet after winning streaks were evaluated using an independent-sample $t$-test to examine the impact of larger wagers on risk preference between the two groups. (2) To evaluate how the outcome of the previous trial influenced participants' risk preferences, a Group (IGD vs. HC) $\times$ Preceding outcome (Win vs. Lose) ANOVA was performed.

Third, to evaluate the group difference in the choice evaluation phase behaviorally, we calculated each participant's ratios of bet versus bank after making a right choice and after making a wrong choice. This index matches with the fMRI analysis for choice evaluation, i.e., right versus wrong choices (as stated in the FMRI data analysis below). Then, a Group (IGD vs. HC) $\times$ Preceding choice (Right vs. Wrong) ANOVA was performed using SPSS statistical software (Version 22.0). Fourth, two general estimating equation (GEE) models with the group (categorical), risk value (continuous) and trial number (continuous) as predictors were constructed using SPSS statistical software
(Version 22.0) to examine the influence of risk value of each trial and group (Model 1) and the interaction (Model 2) on participants' risky choices. The GEE is widely used to model binary outcome variables (i.e., bet vs. bank) with associated residuals (e.g., nested within one participant) through a link function (Liang, Beaty, \& Cohen, 1986; Zeger \& Liang, 1986). In addition, to examine the relationship among task performance, the severity of IGD and reward-related traits, Pearson correlation analyses of only the IGD participants' proportion of bet and their IAT, SSS, BAS/BIS-BAS, and BAS/BIS-BIS scores were performed.

FMRI data analysis. Functional images of all participants were analyzed in the FMRIB Expert Analysis Tool (FEAT) module of FMRIB's Software Library (FSL, v6.0.1, www. fmrib.ox.ac.uk/fsl). First, images were preprocessed as follows: motion correction; removal of nonbrain regions; spatial smoothing (FWHM $=5 \mathrm{~mm}$ ); and high-pass temporal filtering (cutoff $=100 \mathrm{~s}$ ). Second, for images of each participant, customized square waveforms for different explanatory variables (EVs) were constructed and convolved with a double-gamma hemodynamic response function (HRF). Then, statistical contrast maps of interest were generated for further group comparison analysis.

Six EVs were included to examine brain regions responsible for reward anticipation, outcome monitoring and choice evaluation. Specifically, the activity of reward anticipation was modeled based on whether participants chose a risky wheel (bet) or a safe wheel (bank) during the decision stage. The activity of outcome monitoring was modeled based on the outcomes (win or lose) of the risky wheel, which allowed us to evaluate a coarse and rapid processing of reward valence. The activity of choice evaluation was modeled based on whether participants chose right or wrong, i.e., bet-win and bank-lose constituted right choices, whereas bet-lose and bank-win constituted wrong choices. In addition, the dynamic of risk (the risk of the risky wheel varied across trials) may have an effect on the activation pattern of reward anticipation. For each of the two EVs, bet and bank, an additional parametric regressor (i.e., the risk value of the risky wheel on each trial) was included. The parametric values were demeaned before adding to the regressor as modulation values, which guaranteed that the parametric regressor had no correlation with the corresponding constant EV (Poldrack, Mumford, \& Nichols, 2011). Therefore, the main contrasts of interest were (1) bet $>$ bank to dissociate brain regions responsible for anticipating positive reward; (2) win > loss to dissociate regions responsible for monitoring positive outcome; (3) right > wrong to dissociate regions responsible for evaluating positive choice; and (4) the risk effect of bet $>$ bank to dissociate regions responsible for risk assessment.

Third, the generated statistical contrast maps of each participant were registered by their high-resolution T1weighted anatomical image to standard space, yielding images with $2 \times 2 \times 2-\mathrm{mm}^{3}$ spatial resolution. Then, we performed mixed-effect group comparison analyses to obtain group differences (IGD vs. HC) of brain activation during 
the three phases. For the whole brain multiple comparison correction, we used the cluster thresholding in the FEAT module, which is based on Gaussian random field theory (GRFT). This method first thresholds a $Z$-statistical image at a specific $Z$-threshold to define contiguous clusters, and each cluster's estimated significance level (computed by GRFT) is then compared with a cluster probability threshold to show significant clusters. In the present study, for each of the four statistical contrast images, group comparison image was thresholded using cluster-forming correction determined by $Z>2.58$ and a corrected cluster significance threshold of $P<$ 0.05 (Worsley, 2003). Finally, the parameter estimates averaged across all voxels in each cluster showing group differences were extracted from each contrast of interest (e.g., the bet > bank contrast) for each participant as the representative value for brain activation. To identify the relationships among brain activation, the severity of IGD, risk tendency, and reward-related traits, Pearson correlation analyses of only the IGD participants' average parameter estimates and their IAT scores, proportion of bet, SSS, BAS/ BIS-BAS and BAS/BIS-BIS scores were performed.

\section{Ethics}

The study procedures were carried out in conformity to the Declaration of Helsinki. The Institutional Review Board of the Institute of Psychology, Chinese Academy of Sciences approved the current study. All participants provided written informed content and the safety screening scale for MRI scan before the study.

\section{RESULTS}

\section{Questionnaire results}

As shown in Table 1, the BAS-fun seeking score of the IGD group was significantly higher than that of the HC group, indicating a stronger desire for new rewards and willingness to approach a potential reward on the spur of the moment in the IGD group (Carver \& White, 1994).

\section{Behavioral results}

First, the IGD group $(76 \pm 12 \%)$ made significantly more risky choices (bet) than the HC group ((66 $\pm 18 \%), t(51)$ $=2.43, P=0.019$ ) (Fig. $2 \mathrm{~A}$ ), although the mean decision times of the two groups were similar (IGD: $1,330 \pm 234 \mathrm{~ms}$, HC: $1,431 \pm 251 \mathrm{~ms}, t(51)=-1.52, P=0.134)$ (Fig. $2 \mathrm{~B})$. Second, the IGD group had similar winning streaks and risky wheels after winning streaks to those of the $\mathrm{HC}$ group (frequency: IGD: $8.81 \pm 2.66$, HC: $7.69 \pm 3.17, t(51)$ $=1.40, P=0.168$, Fig. $2 \mathrm{C}$; followed risk tendency: IGD: $44.52 \pm 25.60 \%$, HC: $32.24 \pm 25.59 \%, t(51)=1.75, P=$ 0.087, Fig. 2D). For the first ANOVA, the results revealed significant preceding outcomes and group effects (Fig. 2E). Specifically, participants preferred to choose a risky wheel after a loss compared with after a win $(F(1,51)=59.24$, $P<0.001)$. The IGD group chose more risky wheels than the HC group $(F(1,51)=6.04, P=0.017)$. The interaction effect did not reach significance $(F(1,51)=0.63, P=$ $0.433)$.

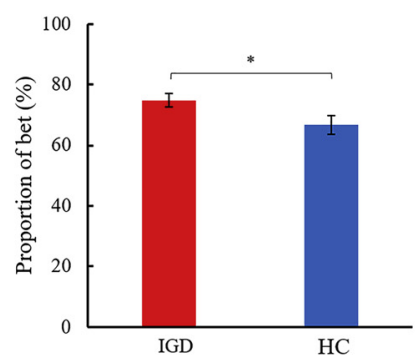

A. Basic Choice

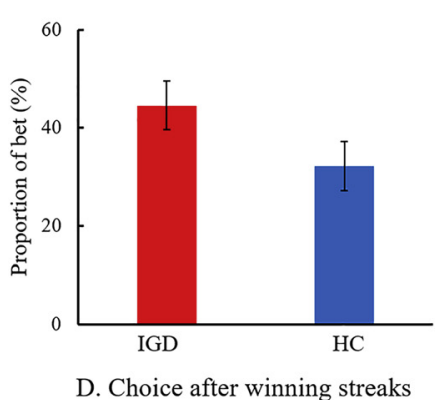

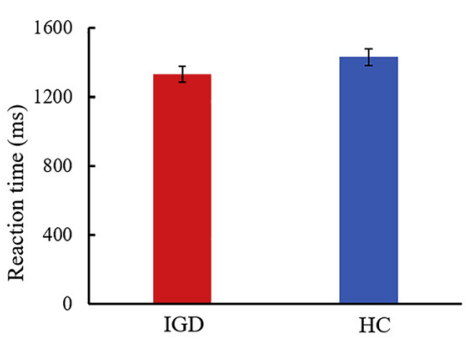

B. Basic Decision Time

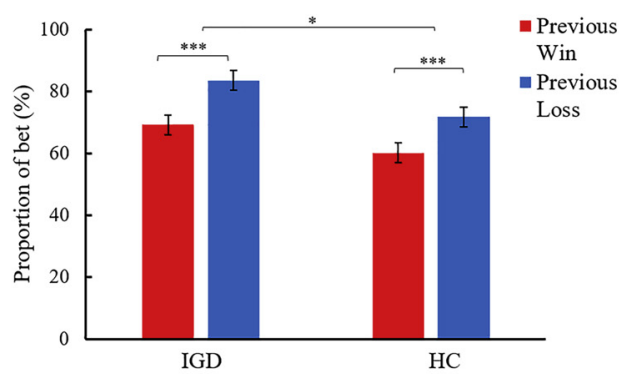

E. Choices after win / loss outcomes
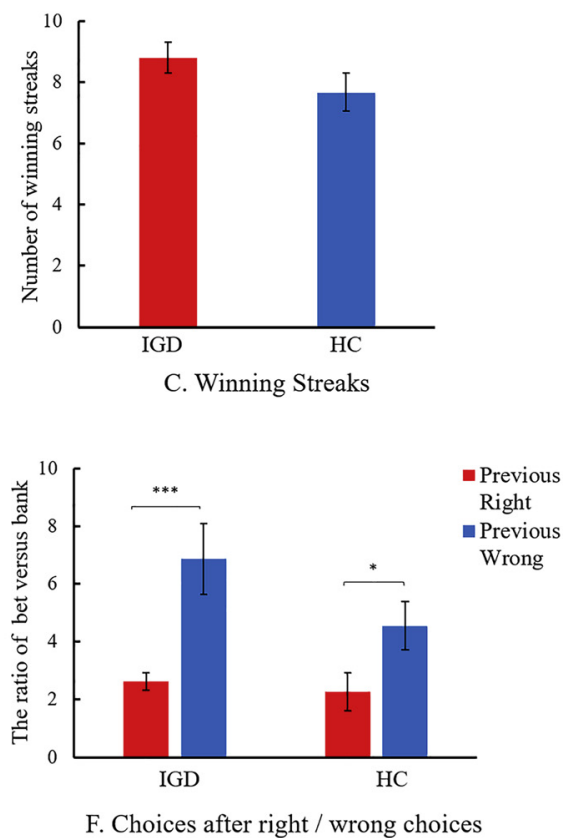

Fig. 2. Behavioral results of the two groups (IGD and HC) in the roulette task. A: The IGD group made significantly more risky choices (bet) than the HC group. B: The mean decision time was similar between the IGD and HC groups. C and D: The IGD group tended to experience more winning streaks and to choose more risky wheels after winning streaks than the HC group; however, statistical significance of this difference was not reached. E: The IGD group made significantly more risky choices than the HC group. F: The IGD and HC groups made similar ratio of bet versus bank, no matter after a right or wrong choice. Abbreviations: IGD = Internet gaming disorder; HC $=$ healthy controls. ${ }^{*} P<0.05,{ }^{* * *} P<0.001$ 
Table 2. GEE models of risk-taking choice predictors in the roulette task

\begin{tabular}{|c|c|c|c|c|c|c|}
\hline \multirow[b]{2}{*}{ Predictors } & \multirow[b]{2}{*}{$\chi^{2}$} & \multirow[b]{2}{*}{$B$} & \multirow[b]{2}{*}{$S E$} & \multicolumn{2}{|c|}{$95 \% \mathrm{CI}$} & \multirow[b]{2}{*}{$P$} \\
\hline & & & & Lower & Upper & \\
\hline \multicolumn{7}{|l|}{ Model 1} \\
\hline Trial number & 0.727 & -0.001 & 0.002 & -0.004 & 0.002 & 0.394 \\
\hline Risk & 0.916 & -0.001 & 0.001 & -0.002 & 0.001 & 0.339 \\
\hline IGD $^{\mathrm{a}}$ & 6.144 & 0.522 & 0.210 & 0.109 & 0.934 & 0.013 \\
\hline \multicolumn{7}{|l|}{ Model 2} \\
\hline Trial number & 0.825 & -0.001 & 0.002 & -0.004 & 0.002 & 0.364 \\
\hline Risk & 0.118 & 0.000 & 0.001 & -0.002 & 0.001 & 0.731 \\
\hline $\mathrm{IGD}^{\mathrm{a}}$ & 8.687 & 0.602 & 0.204 & 0.202 & 1.003 & 0.003 \\
\hline Risk $\times$ IGD $^{\mathrm{a}}$ & 1.654 & -0.001 & 0.001 & -0.004 & 0.001 & 0.198 \\
\hline
\end{tabular}

${ }^{\text {a }}$ Parameter estimates of IGD were computed relative to HC, the parameter for which was therefore redundant. Abbreviations: IGD = Internet gaming disorder; $\mathrm{HC}=$ healthy controls.

Table notes: all models included groups (IGD vs. HC, categorical), risk value (continuous), and trial number (continuous) as predictors. Values in bold present main effects of risk and IGD and the interaction.

Third, for the second ANOVA (Fig. 2F), the preceding choice effect reached significance $(F(1,11.82)=20.27, P<$ $0.001)$, i.e., participants were more likely to bet after a wrong choice than after a right choice. However, the interaction effect $(F(1,11.82)=1.89, P=0.177)$ and group effect $(F(1,44)=2.14, P=0.150)$ did not reach significance. These results indicated that the IGD and HC groups had no significant difference in choice evaluation. Fourth, as shown in Table 2, IGD positively predicted participants' selection of risky choices $(b=0.522, P=0.013)$; moreover, it was not modulated by the risk level of risky wheels, as revealed by the absence of an interaction between group and risk level $(b$ $=-0.001, P=0.198)$. Overall, the present behavioral results revealed a stronger tendency to take risks in the IGD group than in the HC group.
Additionally, the correlational analyses showed that the IGD participants' proportions of bet were significantly correlated with their BAS/BIS-BAS scores $(r=0.603, P=$ 0.000 , FDR corrected), indicating that a higher risk tendency was associated with a stronger desire for a reward. Moreover, we also found a significant positive correlation between the proportion of bet and the BAS-fun seeking scores in the IGD group ( $r=0.590, P=0.001$, FDR corrected).

\section{FMRI results}

As displayed in Fig. 3, both the IGD and HC groups exhibited robust brain activation in reward processingrelated subcortical regions (e.g., NAcc and caudate) during reward anticipation, outcome monitoring and choice

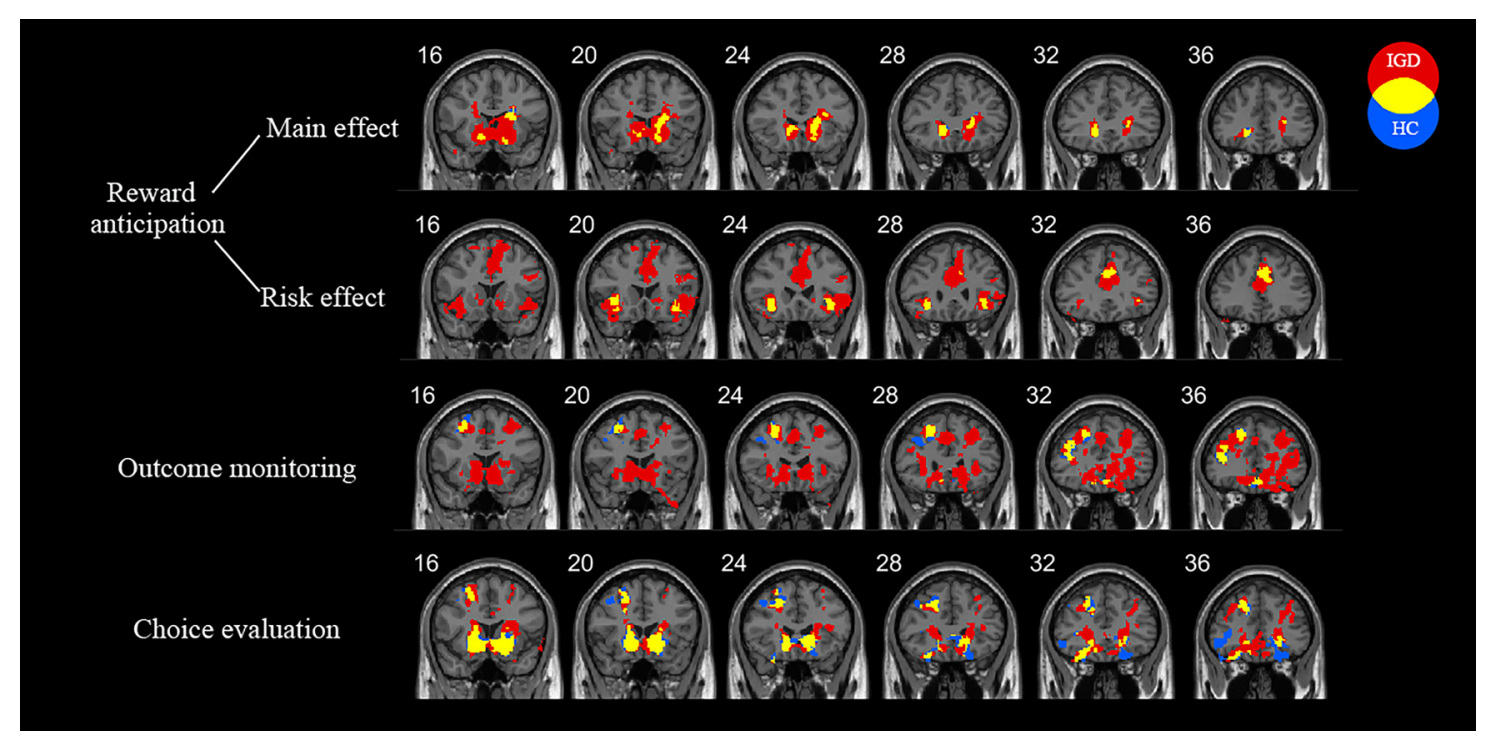

Fig. 3. Brain regions showing significant activation in the two groups (IGD and HC) in the roulette task. Clusters in red indicate the regions activated in the IGD group. Clusters in blue indicate the regions activated in the HC group. Clusters in yellow indicate the regions activated in both groups. Statistical images were corrected using cluster-forming correction determined by $Z>2.58$ and a corrected cluster significance threshold of $P<0.05$, i.e., the threshold of significance for the original $Z$ was 2.58 , and the threshold of significance for the cluster was 0.05 .

IGD $=$ Internet gaming disorder; $\mathrm{HC}=$ healthy controls 


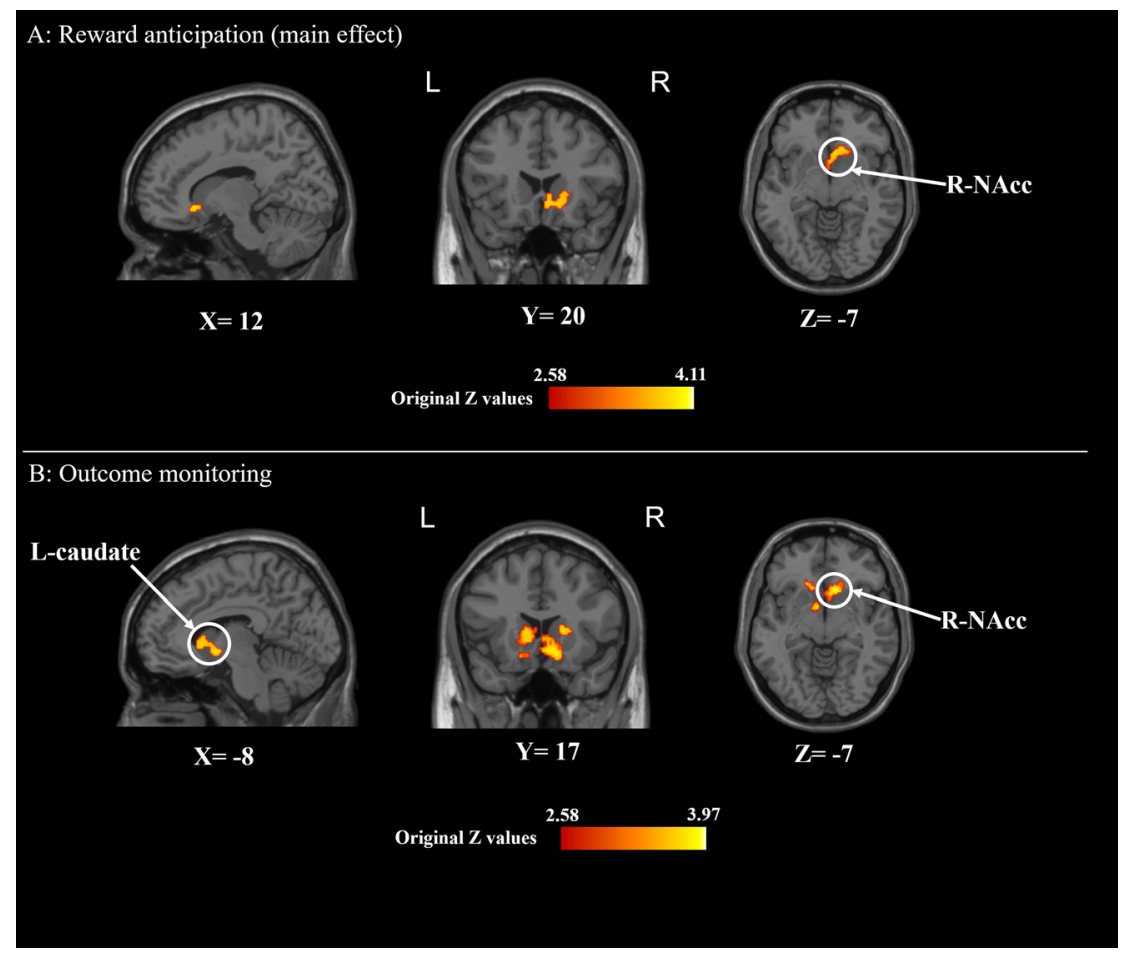

Fig. 4. Brain regions showing significant group differences (IGD vs. HC) in the roulette task. Statistical images were corrected using clusterforming correction determined by $Z>2.58$ and a corrected cluster significance threshold of $P<0.05$. The IGD group showed enhanced brain activities in the R-NAcc in reward anticipation (A) and the R-NAcc and L-caudate in outcome monitoring (B) compared with the HC group. Abbreviations: $\mathrm{IGD}=$ Internet gaming disorder; $\mathrm{HC}=$ healthy controls; $\mathrm{L}=$ left; $\mathrm{R}=$ right; $\mathrm{IAT}=$ Internet addiction test; $\mathrm{NAcc}=$ nucleus accumbens

evaluation phases, as well as robust activation in the ACC and insula during the anticipation phase modulated by the risk values. Figure 4 and Table 3 show the results of the group

Table 3. Brain regions showing significant group differences (IGD vs. HC) in the roulette task

\begin{tabular}{lcccccc}
\hline Region & $x$ & $y$ & $z$ & Size & $Z$ & value \\
\hline $\begin{array}{l}\text { Reward anticipation: IGD }> \\
\quad\end{array}$ & 0 & 0 & -2 & 331 & 4.11 & $0.025^{*}$ \\
$\begin{array}{l}\text { Nucleus } \\
\text { accumbens (R) }\end{array}$ & & & & & & \\
$\begin{array}{l}\text { Outcome monitoring: IGD } \\
\quad \text { Nucleus }\end{array}$ & 10 & 20 & -8 & 414 & 3.97 & $0.004^{*}$ \\
$\begin{array}{l}\text { accumbens (R) } \\
\text { Caudate (L) }\end{array}$ & -8 & 4 & -6 & 287 & 3.62 & $0.029^{*}$ \\
\hline
\end{tabular}

Table notes: we listed the significant clusters of increased (IGD > HC) activation in different phases. Statistical images were corrected using cluster-forming correction determined by $Z>2.58$ and a corrected cluster significance threshold of $P<0.05$. Furthermore, the $P$ values of clusters were corrected using FDR correction for multiple comparison between clusters.

* indicates clusters that pass the FDR correction. Shown are the coordinates of the local maxima in MNI space, the size of the clusters, the Brodmann Area, the maximal $Z$ value and the $P$ value of each cluster. Coordinates represent the local maxima in the corresponding contrast.

Abbreviations: IGD = Internet gaming disorder; $\mathrm{HC}=$ healthy controls; $\mathrm{L}=$ left; $\mathrm{R}=$ right. comparison images. The IGD group exhibited stronger activation in the right NAcc than the $\mathrm{HC}$ group during the reward anticipation phase and stronger right NAcc and bilateral caudate activation during the outcome monitoring phase. Additionally, we conducted a region of interest (ROI) analysis to provide further evidence for the group comparison results. For each of the two group comparison images (i.e., reward anticipation and outcome monitoring phases), clusters that passed the threshold of voxel-level uncorrected $Z>2.58$ were defined as ROIs, and the average parameter estimate values among each of these ROIs were extracted and compared between the IGD and HC groups using an independent-sample $t$-test with the strictest correction method, namely, Bonferroni correction. The ROIs are shown in Fig. 5, and the results are shown in Table 4. The IGD group still had significantly higher activities of the striatum (NAcc and caudate) than the HC group, which was consistent with the results obtained from whole brain correction.

However, for the correlational analyses among the IGD participants' average parameter estimates and their IAT scores, proportion of bet, and SSS, BAS/BIS-BAS and BAS/ BIS-BIS scores, no significant correlation was found. As a supplementary finding, we found that the activation of the right NAcc during the anticipation phase was positively correlated with the SSS scores among all participants $(r=$ $0.279, P=0.043$, uncorrected), indicating that greater activation in the NAcc was associated with a stronger tendency to take risks for novel stimulation. 

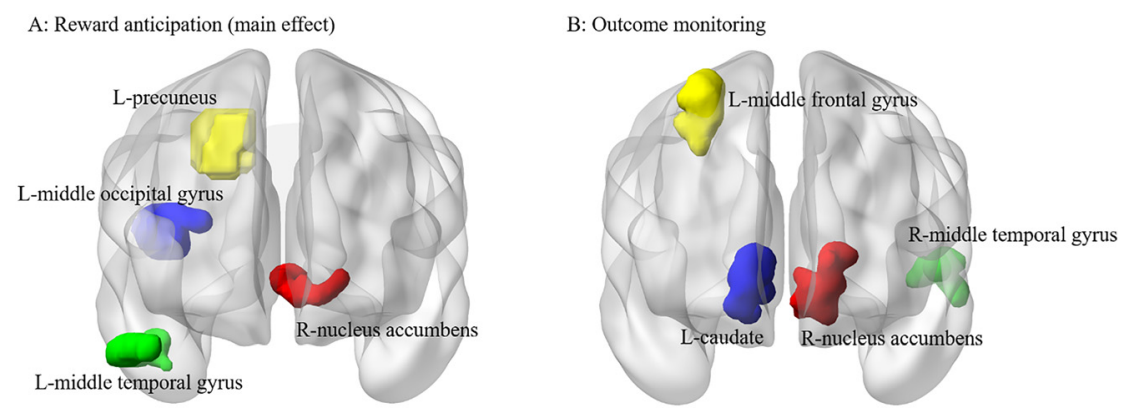

Fig. 5. Regions of interest. For the group comparison images of reward anticipation (A) and outcome monitoring (B), clusters that passed the threshold of voxel-level uncorrected $Z>2.58$ were defined as regions of interest. Abbreviations: $\mathrm{L}$, left; $\mathrm{R}$, right

\section{DISCUSSION}

To the best of our knowledge, this is the first study to simultaneously examine the neural substrates of the three phases of reward processing in individuals with IGD. We found that the IGD group exhibited a higher tendency to take risks in search of potential rewards behaviorally and higher brain activity in reward-related regions (NAcc, caudate) in the reward anticipation and outcome monitoring phases than the HC group. These findings suggested enhanced reward-seeking behavior and specific neural dysfunctions of reward processing (hypersensitivity of striatum) in college students with IGD. Overall, the present study supports the application of the prefrontal-subcortical model of self-regulation in the IGD population and provides specific targets for future research toward effective treatment of IGD.

\section{Reward anticipation}

At the behavioral level, we found that the IGD group chose significantly more risky options than the HC group. Additionally, the ANOVA results revealed that the decision strategies of the two groups were the same, i.e., they both preferred to choose to bet after a loss than after a win. However, the proportion of bet in the IGD group was significantly higher than that in the HC group no matter after wins or losses. The GEE analysis also showed that Internet gaming addiction significantly predicted participants' risky choices. These findings indicated a higher tendency to take risks in the IGD group than in the HC group. Also, we found that the IGD group had a stronger desire and willingness to approach a potential reward, as reflected by the higher BAS-fun seeking scores than in the HC group, and this stronger willingness was associated with a higher proportion of risky choices among the IGD group. All these findings demonstrated that the tendency to take risks in pursuit of potential rewards was stronger in the IGD group than in the HC group, which is consistent with previous research on IGD (Dong \& Potenza, 2016; Li et al., 2019). Moreover, the IGD group's greater risk-taking tendency was associated with a stronger desire for reward, as indicated by the significant correlation between the proportion of bet and BAS total scores in the IGD group.

Additionally, the behavioral results showed that the IGD and HC groups exhibited no significant difference in the number of winning streaks and risky choices after winning streaks despite the relative increase in the number and risky choices in the IGD group. Previous studies also showed that individuals with IGD took a similar degree of risk to $\mathrm{HC}$ on a probability discounting task including risky options with larger amounts and risk values (Li et al., 2016; Tian et al., 2018). This task is widely used to measure individual risktaking behavior and risk calculation ability. Thus, this result

Table 4. Group differences (IGD vs. HC) in regions of interest

\begin{tabular}{|c|c|c|c|c|c|}
\hline Regions of interest & Cluster size & IGD $(N=27)$ & $\mathrm{HC}(N=26)$ & $t$ & $P$ \\
\hline \multicolumn{6}{|l|}{ Reward anticipation phase } \\
\hline Nucleus accumbens (R) & 331 & $17.05 \pm 12.69$ & $2.80 \pm 8.33$ & 4.81 & $0.000^{*}$ \\
\hline Middle occipital gyrus (L) & 248 & $11.19 \pm 14.86$ & $-5.47 \pm 22.41$ & 3.20 & 0.002 \\
\hline Precuneus (L) & 148 & $21.25 \pm 29.17$ & $-1.22 \pm 22.79$ & 3.12 & 0.003 \\
\hline Middle temporal gyrus (L) & 119 & $7.99 \pm 11.64$ & $-0.81 \pm 8.60$ & 3.12 & 0.003 \\
\hline \multicolumn{6}{|l|}{ Outcome monitoring phase } \\
\hline Nucleus accumbens (R) & 414 & $14.94 \pm 11.81$ & $-1.99 \pm 13.29$ & 4.91 & $0.000^{*}$ \\
\hline Caudate (L) & 287 & $18.44 \pm 16.87$ & $0.22 \pm 14.67$ & 4.19 & $0.000^{*}$ \\
\hline Middle frontal gyrus (L) & 239 & $18.26 \pm 37.15$ & $-1.00 \pm 19.22$ & 2.36 & 0.022 \\
\hline Middle temporal gyrus (R) & 200 & $13.24 \pm 30.47$ & $-1.63 \pm 21.69$ & 2.04 & 0.047 \\
\hline
\end{tabular}

Table notes: we listed the clusters that passed the uncorrected threshold at the voxel level $Z>2.58$ in the reward anticipation outcome monitoring phases.

* indicates clusters that passed the Bonferroni correction for multiple comparison.

Abbreviations: IGD = Internet gaming disorder; $\mathrm{HC}=$ healthy controls; $\mathrm{L}=$ left; $\mathrm{R}=$ right. 
may reflect the unabated risk assessment ability of college students with IGD.

At the neural level, during the reward anticipation phase, anticipation of potential gains activated higher right NAcc activity in the IGD group than in the HC group, which was consistent with findings from substance abuse (Becker, Kirsch, Gerchen, Kiefer, \& Kirsch, 2017; Nestor, Hester, \& Garavan, 2010), pathological gambling (Power, Goodyear, \& Crockford, 2012) and Internet addiction (Seok et al., 2015). As a key part of the ventral striatum, the NAcc has long been considered the major player in reward processing because it is the main projection region of the mesolimbic pathway. Many studies have revealed the crucial role of the NAcc in reward anticipation (Knutson, Fong, Adams, Varner, \& Hommer, 2001; Knutson, Westdorp, Kaiser, \& Hommer, 2000; Rademacher et al., 2010). The NAcc showed rewardproportional activation during anticipation of increasing monetary rewards but not punishments (Knutson, Adams, Fong, \& Hommer, 2001), and its activation promoted individuals to approach toward uncertain outcomes (Knutson \& Greer, 2008). Moreover, we found that greater activation in the NAcc was associated with a stronger tendency to take risks for novel stimulation. Accordingly, the present results indicate a strengthened inclination toward potential rewards in the IGD population and thus drive them to make uncertain and risky decisions, which is corroborated by the fact that the IGD group chose risky options over safe options more often than the HC group at the behavioral level.

In particular, in the anticipation phase, we separated brain regions modulated by the risk values of risky options from regions responsible for anticipating potential rewards and found no difference between IGD and HC, i.e., both groups showed similar neural activation (e.g., ACC and insula) in response to the risk values of the risky options. The ACC and insula have commonly been implicated in uncertainty and risk assessment during the decision phase (Critchley, Mathias, \& Dolan, 2001; Knutson, Taylor, Kaufman, Peterson, \& Glover, 2005; Paulus, Rogalsky, Simmons, Feinstein, \& Stein, 2003). The present result suggests that the IGD group may have the same risk assessment ability as the HC group, which is consistent with the present behavioral result concerning risky choices after winning streaks.

\section{Outcome monitoring}

During the outcome monitoring phase, the IGD group exhibited stronger activation for positive outcomes in the right NAcc and bilateral caudate than the HC group. These findings are in accordance with the findings of studies showing that individuals with IGD exhibited greater striatal activity than HC when presented with gains in a cardguessing task (Dong et al., 2017) as well as greater neural responses to addiction-related rewards (i.e., gaming cues) in the striatum during a cue-reactivity task (Ko et al., 2009; Sun et al., 2012) and that individuals with cocaine or alcohol dependence showed increased striatal activity in a monetary incentive delay task (Bjork, Smith, \& Hommer, 2008; Jia et al., 2011). In addition to being involved in reward anticipation, the NAcc has also been suggested to be implicated in the outcome monitoring phase. Both the NAcc and the caudate showed sustained activation after the presentation of positive rewards (Delgado, Nystrom, Fissell, Noll, \& Fiez, 2000; Liu et al., 2007). Additionally, the striatum was found to be associated with craving for gaming in individuals with IGD and to be involved in the processing of addictionrelated rewards (Ko et al., 2009). Therefore, in addition to previous studies revealing enhanced sensitivity to gamingrelated rewards in IGD (Ko et al., 2009; Wang et al., 2017), the present hyperactivation pattern in the striatum for positive outcomes also demonstrated hypersensitivity to general rewards (e.g., monetary rewards) in the IGD population.

\section{Choice evaluation}

During the choice evaluation phase, the IGD and HC groups showed similar behavioral performance and neural activation patterns. As the Group $\times$ Preceding choice ANOVA results showed, both groups adopted the win-stay-lossswitch strategy in the current decision-making task. Specifically, when participants experienced a right choice, the probability of choosing to bet versus bank followed a 'rational' ratio in accordance with the findings in healthy individuals (Liu et al., 2007); however, after a wrong choice, they abandoned this strategy and preferred to bet. At the neural level, both groups exhibited similar neural activation in choice-evaluation-related regions, such as the striatum and OFC. As stated in the introduction section, previous research showed altered frontal-striatal circuit in substance addicts when they were processing $\mathrm{PE}$, one of the cases in choice evaluation (Beylergil et al., 2017; Park et al., 2010; Tanabe et al., 2013). The current results of similar behaviors and neural activation in the IGD and HC groups may demonstrate that the choice evaluation ability in individuals with IGD is still unabated, and they can appropriately evaluate the correctness of their choices by activating the appropriate brain regions for choice evaluation, similar to $\mathrm{HC}$. The use of various strategies to win a game is one of the main features of Internet games, which requires gamers to adapt their next action, shooting, and skill according to the outcome of previous actions. To some degree, individuals with IGD are able to correctly evaluate their decisions. Thus, this may be one reason the IGD group showed no abnormality in the choice evaluation phase. Another possible explanation is that although the current task can examine individual three phases of reward processing, it is a monetary reward task designed for individuals without IGD. The current study applied this task to an IGD population for the first time; thus, it is unclear whether the current task is sensitive enough to capture the characteristics of the IGD population at the choice evaluation phase and the difference between them and healthy people. The development of experimental tasks relevant to Internet games to explore the reward processing of individuals with IGD is needed. 
One limitation of this study should be noted. With regard to the fMRI results of the group comparison with whole brain correction, although we further applied the ROI analysis to support the importance of the striatum in the alteration of IGD, the threshold of voxel-level $Z>2.58$ and cluster-level $P<0.05$ may still have the problem of false positives. The current findings should be interpreted prudently, and future studies with stricter multiple comparison correction and a larger sample size to examine the current results are expected.

\section{CONCLUSIONS}

Using the roulette task, the present study showed enhanced reward-seeking behavior and exaggerated activation of the NAcc and caudate during the reward anticipation and outcome monitoring phases of reward processing but not during the choice evaluation phase in college students with IGD. These results revealed that the enhanced sensitivity of the brain mesolimbic reward system to potential and positive rewards in college students with IGD drives them behaviorally to approach risky options more frequently although they are able to assess the risk values of options and the correctness of decisions properly as HC do. In summary, the present study provides partial support for the application of the prefrontal-subcortical model of self-regulation to the IGD population. Moreover, we identified the specific dysfunctions at different stages of reward processing in IGD, thus enriching the prefrontalsubcortical model of self-regulation and providing specific targets for future research toward effective treatment of IGD.

Funding sources: This work was supported by the National Natural Science Foundation of China under grants number [31571161, 31200782, 31500872, and 31640039]. The funding sources had no involvement in any of the manuscript.

Authors' contribution: LV and QL collected the research data, conducted the statistical analysis and wrote the manuscript. QL and XL designed the study. GY contributed to the statistical analysis. ZL, YZ, and YQ contributed to modifying the manuscript. All authors have approved the final manuscript.

Conflict of interest: The authors declare no conflict of interest.

\section{REFERENCES}

APA (2013). Diagnostic and statistical manual of mental disorders (5th ed.). Washington, DC: American Psychiatric Association. https://doi.org/10.1007/978-3-642-28753-4_1094.

Becker, A., Kirsch, M., Gerchen, M. F., Kiefer, F., \& Kirsch, P. (2017). Striatal activation and frontostriatal connectivity during non-drug reward anticipation in alcohol dependence. Addiction Biology, 22(3), 833-843. https://doi.org/10.1111/adb.12352.

Beylergil, S. B., Beck, A., Deserno, L., Lorenz, R. C., Rapp, M. A., Schlagenhauf, F., et al. (2017). Dorsolateral prefrontal cortex contributes to the impaired behavioral adaptation in alcohol dependence. NeuroImage: Clinical, 15, 80-94. https://doi.org/ 10.1016/j.nicl.2017.04.010.

Bjork, J. M., Brian, K., Fong, G. W., Caggiano, D. M., Bennett, S. M., \& Hommer, D. W. (2004). Incentive-elicited brain activation in adolescents: Similarities and differences from young adults. Journal of Neuroscience, 24(8), 1793-1802. https://doi. org/10.1523/JNEUROSCI.4862-03.2004.

Bjork, J. M., Smith, A. R., \& Hommer, D. W. (2008). Striatal sensitivity to reward deliveries and omissions in substance dependent patients. Neuroimage, 42(4), 1609-1621. https://doi. org/10.1016/j.neuroimage.2008.06.035.

Brand, M., Young, K. S., Laier, C., Wölfling, K., \& Potenza, M. N. (2016). Integrating psychological and neurobiological considerations regarding the development and maintenance of specific Internet-use disorders: An Interaction of Person-AffectCognition-Execution (I-PACE) model. Neuroscience \& Biobehavioral Reviews, 71, 252-266. https://doi.org/10.1016/j. neubiorev.2016.08.033.

Cai, C., Yuan, K., Yin, J., Feng, D., Bi, Y., Li, Y., et al. (2016). Striatum morphometry is associated with cognitive control deficits and symptom severity in internet gaming disorder. Brain Imaging and Behavior, 10(1), 12-20. https://doi.org/10. 1007/s11682-015-9358-8.

Carver, C. S., \& White, T. L. (1994). Behavioral inhibition, behavioral activation, and affective responses to impending reward and punishment: The BIS/BAS Scales. Journal of Personality \& Social Psychology, 67(2), 319-333. https://doi.org/10. 1037/0022-3514.67.2.319.

Cohen, M. X. (2006). Individual differences and the neural representations of reward expectation and reward prediction error. Social Cognitive and Affective Neuroscience Electronic Resource, 2(1), 20-30. https://doi.org/0.1093/scan/nsl021.

Cohen, M. X., \& Ranganath, C. (2005). Behavioral and neural predictors of upcoming decisions. Cognitive, Affective, \& Behavioral Neuroscience, 5(2), 117-126. https://doi.org/10. 3758/CABN.5.2.117.

Cooper, S., Robison, A. J., \& Mazei-Robison, M. S. (2017). Reward circuitry in addiction. Neurotherapeutics. https://doi.org/10. 1007/s13311-017-0525-z.

Critchley, H. D., Mathias, C. J., \& Dolan, R. J. (2001). Neural activity in the human brain relating to uncertainty and arousal during anticipation. Neuron, 29(2), 537-545. https://doi.org/10. 1016/S0896-6273(01)00225-2.

Delgado, M. R., Nystrom, L. E., Fissell, C., Noll, D., \& Fiez, J. A. (2000). Tracking the hemodynamic responses to reward and punishment in the striatum. Journal of Neurophysiology, 84(6), 3072-3077. https://doi.org/10.1152/jn.2000.84.6.3072.

Dong, G., Huang, J., \& Du, X. (2011). Enhanced reward sensitivity and decreased loss sensitivity in internet addicts: An fMRI study during a guessing task. Journal of Psychiatric Research, 45(11), 1525-1529. https://doi.org/10.1016/j.jpsychires.2011.06.017.

Dong, G., Li, H., Wang, L., \& Potenza, M. (2017). Cognitive control and reward/loss processing in Internet gaming disorder: Results 
from a comparison with recreational Internet game-users. $E u$ ropean Psychiatry, 44, 30-38. https://doi.org/10.1016/j.eurpsy. 2017.03.004.

Dong, G., \& Potenza, M. N. (2016). Risk-taking and risky decisionmaking in Internet gaming disorder: Implications regarding online gaming in the setting of negative consequences. Journal of Psychiatric Research, 73(1), 1-8. https://doi.org/10.1016/j. jpsychires.2015.11.011.

Dong, G., Wang, Z., Wang, Y., Du, X., \& Potenza, M. N. (2019). Gender-related functional connectivity and craving during gaming and immediate abstinence during a mandatory break: Implications for development and progression of internet gaming disorder. Progress In Neuro-Psychopharmacology \& Biological Psychiatry, 88, 1-10. https://doi.org/10.1016/j.pnpbp. 2018.04.009.

Gardner, E. L. (2011). Addiction and brain reward and Antireward pathways. Advances in Psychosomatic Medicine, 30, 22. https:// doi.org/10.1159/000324065.

Hare, T. A., O"Doherty, J., Camerer, C. F., Schultz, W., \& Rangel, A. (2008). Dissociating the role of the orbitofrontal cortex and the striatum in the computation of goal values and prediction errors - supplemental data. Journal of Neuroscience, 28(22), 5623-5630. https://doi.org/10.1523/JNEUROSCI.130908.2008 .

Heatherton, T. F., \& Wagner, D. D. (2011). Cognitive neuroscience of self-regulation failure. Trends in Cognitive Sciences, 15(3), 132-139. https://doi.org/10.1016/j.tics.2010.12.005.

Jia, Z., Worhunsky, P. D., Carroll, K. M., Rounsaville, B. J., Stevens, M. C., Pearlson, G. D., et al. (2011). An initial study of neural responses to monetary incentives as related to treatment outcome in cocaine dependence. Biological Psychiatry, 70(6), 553-560. https://doi.org/10.1016/j.biopsych.2011.05.008.

Kerstin, P., Peter, B., \& Quartz, S. R. (2006). Neural differentiation of expected reward and risk in human subcortical structures. Neuron, 51(3), 381-390. https://doi.org/10.1016/j.neuron.2006. 06.024 .

Knutson, B., Adams, C. M., Fong, G. W., \& Hommer, D. (2001). Anticipation of increasing monetary reward selectively recruits nucleus accumbens. Journal of Neuroscience, 21(16), 15. https:// doi.org/10.0000/PMID11459880.

Knutson, B., Fong, G. W., Adams, C. M., Varner, J. L., \& Hommer, D. (2001). Dissociation of reward anticipation and outcome with event-related fMRI. Neuroreport, 12(17), 3683. https://doi. org/10.1097/00001756-200112040-00016.

Knutson, B., \& Greer, S. M. (2008). Anticipatory affect: Neural correlates and consequences for choice. Philosophical Transactions of the Royal Society of London, 363(1511), 3771-3786. https://doi.org/10.1098/rstb.2008.0155.

Knutson, B., Taylor, J., Kaufman, M., Peterson, R., \& Glover, G. (2005). Distributed neural representation of expected value. Journal of Neuroscience, 25(19), 4806-4812. https://doi.org/10. 1523/JNEUROSCI.0642-05.2005.

Knutson, B., Westdorp, A., Kaiser, E., \& Hommer, D. (2000). FMRI visualization of brain activity during a monetary incentive delay task. Neuroimage, 12(1), 20-27. https://doi.org/10.1006/nimg. 2000.0593.

Ko, C., Ju-Yu, Y., Sue-Huei, C., \& Peng-Wei, C., et al. (2014). Evaluation of the diagnostic criteria of Internet gaming disorder in the DSM-5 among young adults in Taiwan. Journal of Psychiatric Research, 53, 103-110. https://doi.org/ 10.1016/j.jpsychires.2014.02.008.

Ko, C., Liu, G., Hsiao, S., Yen, J., Yang, M., Lin, W., et al. (2009). Brain activities associated with gaming urge of online gaming addiction. Journal of Psychiatric Research, 43(7), 739. https:// doi.org/10.1016/j.jpsychires.2008.09.012.

Kuss, D. J., \& Griffiths, M. D. (2012). Internet gaming addiction: A systematic Review of empirical research. International Journal of Mental Health \& Addiction, 10(2), 278-296. https://doi.org/ 10.1007/s11469-011-9318-5.

Lai, C. M., Mak, K. K., Watanabe, H., Ang, R. P., Pang, J. S., \& Ho, R. C. (2013). Psychometric properties of the internet addiction test in Chinese adolescents. Journal of Pediatric Psychology, 38(7), 794-807. https://doi.org/10.1093/jpepsy/ jst022.

Liang, K., Beaty, T., \& Cohen, B. (1986). Application of odds ratio regression models for assessing familial aggregation from casecontrol studies. American Journal of Epidemiology, 124(4), 678683. https://doi.org/10.1007/BF00935358.

Li, Q., Tian, M., Taxer, J., Zheng, Y., Wu, H., Sun, S., et al. (2016). Problematic internet users' discounting behaviors reflect an inability to delay gratification, not risk taking. Cyberpsychology, Behavior, and Social Networking, 19(3), 172-178. https://doi. org/10.1089/cyber.2015.0295.

Liu, X., Hairston, J., Schrier, M., \& Fan, J. (2011). Common and distinct networks underlying reward valence and processing stages: A meta-analysis of functional neuroimaging studies. Neuroscience \& Biobehavioral Reviews, 35(5), 1219-1236. https://doi.org/10.1016/j.neubiorev.2010.12.012.

Liu, X., Powell, D. K., Wang, H., Gold, B. T., Corbly, C. R., \& Joseph, J. E. (2007). Functional dissociation in frontal and striatal areas for processing of positive and negative reward information. Journal of Neuroscience, 27(17), 4587-4597. https://doi. org/10.1523/JNEUROSCI.5227-06.2007.

Liu, L., Yip, S. W., Zhang, J. T., Wang, L. J., Shen, Z. J., Liu, B., et al. (2017). Activation of the ventral and dorsal striatum during cue reactivity in Internet gaming disorder. Addiction Biology, 22(3), 791-801. https://doi.org/10.1111/adb.12338.

Li, Q., Wang, Y., Yang, Z., Dai, W., Zheng, Y., Sun, Y., et al. (2019). Dysfunctional cognitive control and reward processing in adolescents with Internet gaming disorder. Psychophysiology, e13469. https://doi.org/10.1111/psyp.13469.

Li, Y., Zhang, Y., Jiang, Y., Li, H., Mi, S., Yi, G., et al. (2008). The Chinese version of the BIS/BAS scale:Reliability and validity. Community Mental Health Journal. https://doi.org/10.3724/SP. J.1041.2008.00418.

Nestor, L., Hester, R., \& Garavan, H. (2010). Increased ventral striatal BOLD activity during non-drug reward anticipation in cannabis users. Neuroimage, 49(1), 1133-1143. https://doi.org/ 10.1016/j.neuroimage.2009.07.022.

Park, S. Q., Kahnt, T., Beck, A., Cohen, M. X., Dolan, R. J., Wrase, J., et al. (2010). Prefrontal cortex fails to learn from reward prediction errors in alcohol dependence. Journal of Neuroscience, 30(22), 7749-7753. https://doi.org/10.1523/JNEUROSCI. 5587-09.2010.

Paulus, M. P., Rogalsky, C., Simmons, A., Feinstein, J. S., \& Stein, M. B. (2003). Increased activation in the right insula during 
risk-taking decision making is related to harm avoidance and neuroticism. Neuroimage, 19(4), 1439-1448. https://doi.org/10. 1016/S1053-8119(03)00251-9.

Pawlikowski, M., Altstötter-Gleich, C., \& Brand, M. (2013). Validation and psychometric properties of a short version of Young's Internet Addiction Test. Computers in Human Behavior, 29(3), 1212-1223. https://doi.org/10.1016/j.chb.2012.10.014.

Petry, N. M., Rehbein, F., Gentile, D. A., Lemmens, J. S., Rumpf, H. J., Mößle, T., et al. (2014). An international consensus for assessing internet gaming disorder using the new DSM-5 approach. Addiction, 109(9), 1399-1406. https://doi.org/10.1111/add.12457.

Poldrack, R. A., Mumford, J. A., \& Nichols, T. E. (2011). Handbook of functional MRI data analysis. England: Cambridge University Press. https://doi.org/10.1017/CBO9780511895029.

Power, Y., Goodyear, B., \& Crockford, D. (2012). Neural correlates of pathological gamblers preference for immediate rewards during the Iowa gambling task: An fMRI study. Journal of Gambling Studies, 28(4), 623-636. https://doi.org/10.1007/ s10899-011-9278-5.

Rademacher, L., Krach, S., Kohls, G., Irmak, A., Gründer, G., \& Spreckelmeyer, K. N. (2010). Dissociation of neural networks for anticipation and consumption of monetary and social rewards. Neuroimage, 49(4), 3276-3285. https://doi.org/10.1016/j. neuroimage.2009.10.089.

Ramnani, N., Elliott, R., Athwal, B. S., \& Passingham, R. E. (2004). Prediction error for free monetary reward in the human prefrontal cortex. Neuroimage, 23(3), 777-786. https://doi.org/10. 1016/j.neuroimage.2004.07.028.

Report on China's game industry from January to June 2019 (2019). China Audio-visual and digital publishing association game publishing committee \& international data corporation. Retrieved from http://www.cnr.cn/hn/jrhn/20191219/ t20191219_524905431.shtml.

Schultz, W. (2004). Neural coding of basic reward terms of animal learning theory, game theory, microeconomics and behavioural ecology. Current Opinion in Neurobiology, 14(2), 139-147. https://doi.org/10.1016/j.conb.2004.03.017.

Schultz, W., Dayan, P., \& Montague, P. R. (1997). A neural substrate of prediction and reward. Science, 275(5306), 1593-1599. https://doi.org/10.1126/science.275.5306.1593.

Seok, J.-W., Lee, K. H., Sohn, S., \& Sohn, J.-H. (2015). Neural substrates of risky decision making in individuals with Internet addiction. Australian \& New Zealand Journal of Psychiatry, 49(10), 923-932. https://doi.org/10.1177/0004867415598009.

Sun, Y., Huang, Y., Seetohul, R. M., Wang, X., Zheng, Y., Li, Q., et al. (2012). Brain fMRI study of crave induced by cue pictures in online game addicts (male adolescents). Behavioural Brain Research, 233(2), 563. https://doi.org/10.1016/j.bbr.2012.05.005.

Tanabe, J., Reynolds, J., Krmpotich, T., Claus, E., Thompson, L. L., Du, Y. P., et al. (2013). Reduced neural tracking of prediction error in substance-dependent individuals. American Journal of Psychiatry, 170(11), 1356-1363. https://doi.org/10.1176/appi. ajp.2013.12091257.

Tian, M., Tao, R., Zheng, Y., Zhang, H., Yang, G., Li, Q., et al. (2018). Internet gaming disorder in adolescents is linked to delay discounting but not probability discounting. Computers in Human Behavior, 80, 59-66. https://doi.org/10.1016/j.chb. 2017.10.018.

Volkow, N. D., Wang, G.-J., Fowler, J. S., Tomasi, D., \& Telang, F. (2011). Addiction: Beyond dopamine reward circuitry. Proceedings of the National Academy of Ences, 108(37). https://doi. org/10.1073/pnas.1010654108.

Wang, W., Wu, Y. X., Peng, Z. G., Lu, S. W., Yu, L., Wang, G. P., et al. (2000). Test of sensation seeking in a Chinese sample. Personality \& Individual Differences, 28(1), 169-179. https:// doi.org/10.1016/S0191-8869(99)00092-6.

Wang, L., Wu, L., Wang, Y., Li, H., Liu, X., Du, X., et al. (2017). Altered brain activities associated with craving and cue reactivity in people with internet gaming disorder: Evidence from the comparison with recreational internet game users. Frontiers in Psychology, 8, 1-12. https://doi.org/10.3389/fpsyg.2017. 01150 .

Widyanto, L., \& Mcmurran, M. (2004). The psychometric properties of the internet addiction test. Cyberpsychology \& Behavior the Impact of the Internet Multimedia \& Virtual Reality on Behavior \& Society, 7(4), 443. https://doi.org/10.1089/cpb.2004. 7.443.

Worsley, K. J. (2003). Detecting activation in fMRI data. Statal Methods in Medical Research, 12(5), 401-418. https://doi.org/ 10.1191/0962280203sm340ra.

Xing, L., Yuan, K., Bi, Y., Yin, J., Cai, C., Feng, D., et al. (2014). Reduced fiber integrity and cognitive control in adolescents with internet gaming disorder. Brain Research, 1586, 109-117. https://doi.org/10.1016/j.brainres.2014.08.044.

Yao, Y., Chen, P., Li, C. S. R., Hare, T. A., Li, S., Zhang, J., et al. (2017). Combined reality therapy and mindfulness meditation decrease intertemporal decisional impulsivity in young adults with Internet gaming disorder. Computers in Human Behavior, 68(5), 210-216. https://doi.org/10.1016/j.chb.2016.11.038.

Young, K. (1998). Caught in the net: How to recognize the signs of internet addiction-and a winning strategy for recovery. New York: John Wiley \& Sons. https://doi.org/10.1177/ 1073191114528570.

Yuan, K., Yu, D., Cai, C., Feng, D., Li, Y., Bi, Y., et al. (2016). Frontostriatal circuits, resting state functional connectivity and cognitive control in internet gaming disorder. Addiction Biology, 22(3). https://doi.org/10.1111/adb.12348.

Zeger, S. L., \& Liang, K.-Y. (1986). Longitudinal data analysis for discrete and continuous outcomes. Biometrics, 42(1), 121-130. https://doi.org/10.2307/2531248.

Open Access. This is an open-access article distributed under the terms of the Creative Commons Attribution-NonCommercial 4.0 International License (https:// creativecommons.org/licenses/by-nc/4.0/), which permits unrestricted use, distribution, and reproduction in any medium for non-commercial purposes, provided the original author and source are credited, a link to the CC License is provided, and changes - if any - are indicated. 